\title{
Importance of remission and residual somatic symptoms in health-related quality of life among outpatients with major depressive disorder: a cross-sectional study
}

Jong-Min Woo ${ }^{1,2}$, Hong Jin Jeon ${ }^{3,4}$, Eunsun Noh ${ }^{5,6}$, Hyo-Jin Kim7, Sun Woo Lee ${ }^{8}$, Kyung Kyu Lee ${ }^{9}$, Sung Hwan Kim ${ }^{10}$ and Jin Pyo Hong ${ }^{11^{*}}$

\begin{abstract}
Background: Major depressive disorder (MDD) is strongly associated with an impaired quality of life (QoL), which is itself affected by various factors. Symptom-oriented ratings poorly reflect the impact of disease on the QoL and level of functioning of the mental health of subjects. The purpose of this study was to assess health-related QoL (HRQOL) using preference-based measures in outpatients with MDD with regard to their remission achievement and clinical factors affecting the HRQoL.
\end{abstract}

Methods: This was a cross-sectional observational study. We recruited 811 patients with MDD from 14 psychiatric outpatient clinics in Korea. They were divided into three groups as follows: a new visit group ( $n=287)$, a remitted group ( $n=235)$, and a non-remitted group $(n=289)$. The 17-item Hamilton Depression Rating Scale was used to assign patients to the remitted or non-remitted group. The general HRQoL was assessed with the EuroQol 5D (EQ-5D), using both the EQ-5D index score and the EuroQol Visual Analog Scale (EQ-VAS). The disease-specific HRQoL was assessed with the Quality of Life Enjoyment and Satisfaction Questionnaire Short Form (Q-LES-Q-SF).

Results: The non-remitted group showed a significant impairment of HRQOL in view of the subscales of EQ-5D index scores, EQ-VAS, and Q-LES-Q-SF. The EQ-5D index score in the remitted group was 0.77 0.10, while it was 0.570 .23 in the non-remitted group and 0.580 .24 in the new visit group $(p<0.0001)$. The EQ-VAS scores for the remitted and non-remitted groups were 72.516 .6 and 50.920 .3 , respectively $(p<0.0001)$. Likewise, patients with remission had the Q-LES-Q-SF total score of 46.5 8.8, whereas tho se with non-remission reported $36.77 .7(p<0.0001)$. The symptom severity measured by the Depression and Somatic Symptoms Scale was significantly correlated with the HRQoL. Furthermore, patients with severe somatic symptoms showed a significantly lower EQ-5D index score (0.54 0.24) than those with mild/moderate somatic symptoms $(0.750 .12 ; p=0.002)$.

Conclusion: Non-remitted MDD patients, especially those with more severe somatic symptoms, show a distinct impairment of HRQOL and more clinical symptoms, suggesting the importance of achieving remission in the treatment of MDD.

Keywords: Major depressive disorder, Quality of life, EQ-5D, Q-LES-Q-SF

\footnotetext{
* Correspondence: jphong@amc.seoul.kr

${ }^{11}$ Department of Psychiatry, Asan Medical Center, Ulsan University College of Medicine, 388-1 Pungnap-2dong, Songpa-gu, Seoul 138-736, South Korea Full list of author information is available at the end of the article
} 


\section{Introduction}

Major depressive disorder (MDD) is strongly associated with an impaired quality of life (QoL) and several studies have investigated the factors affecting this relationship [1-3].

Health related QoL (HRQoL) covers a wide range of dimensions including psychological status, functional abilities, subjective well-being, social interactions, role performance and physical health [4]. Due to this nature of HRQoL, assessment of HRQoL has received considerable research attention and provided potential for a more comprehensive evaluation of treatment outcomes.

However, symptom-oriented ratings poorly reflect the impact of mental health on QoL and level of functioning. Thus, QoL has been mainly assessed with patientreported outcome (PRO) instruments. Recently, the importance of PRO measures in clinical trials for new drug, biologic agents and devices was underscored by the release of the US Food and Drug Administration's draft guidance for industry $[5,6]$.

Recently, results of the Factors Influencing Depression Endpoints Research (FINDER) study of the European Union demonstrated that antidepressant treatment was associated with an improved HRQoL and that clinical factors, including the presence of somatic and painful symptoms, were negatively associated with HRQoL $[1,7,8]$. Depressed patients who fail to obtain full remission, which is defined as almost full recovery of depression symptoms to the level of a person without depression, show a higher risk of being depressed again and continue to experience functional impairment [9]. These results suggest that achieving remission and severity of certain residual symptoms can be more important for improving QoL.

Previous studies suggested an impaired HRQoL in patients with depression and an improved HRQoL in treated patients; however, the relationship between HRQoL, particularly measured using PRO and remission status is less well understood. In our current study, we quantified both generic and disease-specific measures of QoL and then compared them among three subgroups (new visit, remitted, and non-remitted groups). In addition, we examined the effect of HRQoL on suicidal thoughts and suicide attempts. We hypothesized that the non-remitted group would show more severe impairment in QoL than the new visit group and remitted group and, moreover, that the severity of somatic and pain symptoms would be closely associated with both generic and disease-specific QoL measures.

\section{Methods}

\section{Subjects}

The data of Korean patients with MDD were derived from the Korean Burden of Illness Study. This study was a noninterventional, multicenter-based, naturalistic observational, cross-sectional, and outpatient-based study of patients with MDD. All patients were enrolled from 14 regional psychiatric outpatient clinics in Korea. The study was conducted between December 2011 and September 2012. All study procedures were approved by the Institutional Review Board of Asan Medical Center and other sites. Written informed consent was obtained from all study patients before study initiation.

\section{Inclusion and exclusion criteria}

Patients 18 years or older and meeting the DSM-IV criteria for a single or recurrent non-psychotic MDD were included. Each consecutive patient was evaluated according to the inclusion and exclusion criteria and was then allocated to one of three groups: (1) a new visit group, (2) a remitted group (17-item Hamilton Depression Rating Scale [HAM-D-17] <8), or (3) a non-remitted group (HAM-D-17 $\geq 8$ ). Each hospital was allowed to consecutively allocate approximately eight patients to each group. Groups 2 and 3 included patients who had received MDD treatment for less than 6 months in order to exclude any bias possibility in calculating the lost productivity costs due to decreased social occupational function related to other emotional problems.

Exclusion criteria included history of bipolar disorder, schizophrenia, schizoaffective disorder, psychosis, anorexia or bulimia nervosa, obsessive-compulsive disorder, or a serious general medical condition.

\section{Measurement of depressive symptoms}

The 17-item HAM-D was used to measure the severity of depressive symptoms at each visit [10]. The HAM-D-17 is a clinician-administered depression assessment scale, evaluating mood and vegetative symptoms [10]. Each item was rated with a score of 0-4 (where the scores are equivalent to absent, doubtful/trivial, mild, moderate and severe) or 02 (absent, doubtful/mild, and obvious, distinct/severe) [10]. By summing the score of each item, the total scores for HAM-D-17 can range from 0 to 54. In general, a total HAM-D-17 score of 7 or less is accepted as an indicator of remission [11]. It has been reported an internal consistency of 0.83 [12], inter-rater reliability of 0.97 [13], and testretest reliability of 0.81 [14], showing adequate reliability.

\section{Measurement of somatic symptoms}

The Depression and Somatic Symptoms Scale (DSSS) was applied to measure somatic symptoms in MDD patients [15]. The DSSS is a 22-item self-administered rating scale containing three subscales: a Depression Subscale (DS), a Pain Subscale (PS), and a Somatic Subscale (SS). The DS has 12 items, including three vegetative symptoms and fatigue, whereas the SS has 10 items, including five pain items composed of the 5-item PS. Each item was rated with a score of 0 3: 0 (not at all), 1 (mild), 2 (moderate), 
or 3 (severe). Thus, the range of summed scores was 066 . The scale shows a good validity and reliability, with higher scores indicating stronger symptoms. The Korean Version of DSSS has a Cronbach's alpha of 0.90 and shows a relatively high test-retest reliability $(\mathrm{r}=0.83, \mathrm{p}<0.01)[16,17]$.

\section{Measures of QoL}

In order to assess HRQoL, we used generic instruments, including the European Quality of Life-5 Dimensions (EQ-5D; containing an index score and visual analog scale [VAS]), and disease- or condition-specific QoL measures, including the Quality of Life Enjoyment and Satisfaction Questionnaire (Q-LES-Q).

\section{EuroQol (EQ-5D and EQ-VAS)}

EQ-5D is a standardized preference-based measure of health status developed by the EuroQol Group that is applicable to mental health conditions such as mild to moderate depression and anxiety [18-20]. The EQ-5D was developed to provide a simple, generic measure of health for clinical and economic appraisals [20]. It provides a simple descriptive profile and a single index value for health status that can be used in the clinical and economic evaluation of health care as well as in population health surveys. The EQ-5D is designed for self-completion by respondents and essentially consists of two pages. The EQ-5D descriptive system comprises the following five dimensions (with three levels) and the EQ visual analog scale (EQ-VAS). The EQ-5D consists of five dimensions (mobility, self-care, usual activities, pain/discomfort, anxiety/depression), each with three levels of severity in health utility (no problems/some or moderate problems/extreme problems) recording an individuals ratings of EQ-5D health states. The Korean version of the EQ-5D was developed and validated by Kim et al. in 2005 [21]. The EQ-5D index score was calculated based on the weights elicited from a large national survey of the Korean population [22].

\section{The Q-LES-Q}

The Q-LES-Q is a self-report instrument designed to measure satisfaction and enjoyment in various domains of functioning (e.g., physical health, work, and household duties) [23]. It can be applied to depressed individuals [24]. Many studies have used the Q-LES-Q to measure life satisfaction and enjoyment in patients with depression during pre- and post-treatment phases [23,25,26]. The Q-LES-Q Short Form (Q-LES-Q-SF) used in this study consists of 16 items, of which the first 14 assess discrete domains such as social relationships, living or housing situation, and physical health. Item 15 concerns the respondents satisfaction with medication they are taking, if applicable. Item 16 is a global rating in which respondents are asked to rate their overall life satisfaction and contentment. Each item is scored on a 5-point Likert scale that indicates the degree of enjoyment or satisfaction achieved during the past week ( 1 = very poor, $5=$ very good $)$. Higher scores indicate better function. Items 1 through 14 of the Q-LES-Q-SF can be summed to obtain a total score, with higher scores indicating greater life enjoyment and satisfaction. For the present analyses, we used the total score generated by the sum of the first 14 items. This scale has a Cronbachs alpha of 0.90 and a test-retest reliability of 0.74 [23]. The construct validity of the Q-LES-Q is supported by moderately negative correlations with the Clinical Global Impressions Severity of Illness scale (CGI-S) $(r=0.62$ for the summary scale) and the 17-item HAM-D, compared in a depressed population [23]. To compare with other instruments of HRQoL we also obtained the Q-LES-Q index score by converting the Q-LES-Q total score to a percentage.

\section{Measures of suicidal thoughts and suicide attempts}

We assessed suicide attempts and suicidal thoughts during the past month before study inclusion using the Korean version of the Mini-International Neuropsychiatric Interview (MINI) suicidality module [27]. The MINI suicidality module is composed of six questions including wish for death, wish for self-harm, suicidal thoughts, suicide plans, suicide attempts in the past month, and lifetime suicide attempts. We included the Mini-C2 (suicide attempts in the past month) and Mini-C3 (suicidal thoughts) in the analysis to examine the association between HRQoL and suicidal thoughts/suicide attempts.

\section{Statistical analysis}

Descriptive statistics (mean, standard deviation) were obtained for continuous data in each MDD patient group. ANOVA (or Kruskal-Wallis test) was performed to compare the patient groups. Tukeys multiple comparison method was used for post-hoc group comparisons after the significance for ANOVA was determined. The QoL was compared between the MDD patient groups using ANCOVA, controlling for the depression symptom score as covariate. The association between the QoL and depression symptoms score was evaluated by stepwise regression using the full set of variables of the DSSS adjusted by age and sex. Additionally, the association between suicidal thoughts/suicide attempts and HRQoL using logistic regression analysis was adjusted by age and DSSS score. Frequency and percentage were obtained for categorical data and the chi-square test was applied to the categorical data. All analyses were performed using SAS version 9.2 (SAS Institute, Cary, NC).

\section{Results}

Baseline characteristic of the study subjects

A total of 811 patients with MDD were included in this study, 235 in the remitted group, 289 in the non-remitted 
group, and 287 in the new visit group. The demographics and clinical characteristics of study subjects are presented in Table 1 . Approximately $70 \%$ of the patients were women in each group and the mean age ranged from 44.4 to 47.6 years across the groups. There were $62.3 \%$ unemployed subjects in the non-remitted group while the remitted group showed an unemployment rate of $57.4 \%$. In addition, patients in the non-remitted group demonstrated a slightly higher number of depressive episodes than the patients in the remitted group (mean standard deviation [SD], 1.3 2.2 vs. 1.72 .6 ) and a slightly longer duration of MDD (7.3 16.6 vs. 8.120 .5 months).

\section{Disease severity of the subjects}

Table 2 shows the symptom severity measured by $17-$ item HAM-D scores and DSSS. Patients who achieved remission showed a HAM-D score of 4.52 .1 , which is significantly lower than the scores of the non-remitted group (16.6 5.8) and the new visit group (17.8 7.0) $(\mathrm{p}<0.0001)$. Similarly, the total scores of depressive and somatic symptoms were significantly lower in the remitted group than in the non-remitted and new visit group.
The depressive symptom scores in the remitted group were 5.95 .3 , whereas they were 16.87 .2 in the nonremitted group and 17.4 7.2 in the new visit group ( $\mathrm{p}<0.0001)$. In addition, the total score of somatic symptoms including pain was 4.0 3.9 in the remitted group, 10.66 .2 in the non-remitted group, and 10.95 .8 in the new visitors $(\mathrm{p}<0.0001)$.

\section{HRQoL}

The HRQoL measured by the EQ-5D index and EQVAS and the disease-specific QoL measured by the QLES-Q-SF are described in Table 3. The EQ-5D index score was 0.770 .10 in the remitted group and 0.57 0.23 in the non-remitted group $(\mathrm{p}<0.0001)$. Pain/discomfort and anxiety/depression were the two strongest influencers of patients' HRQoL among each dimension of EQ-5D. Approximately 65\% of patients in remission reported no problems with pain/discomfort, whereas more than $66 \%$ of patients in the non-remitted group reported some/severe problems with pain/discomfort. Similar to the pain/discomfort domain, approximately $61 \%$ of the remitted group reported no problems with

Table 1 Demographics of the study subjects in the new visit, remitted, and non-remitted MDD groups

\begin{tabular}{|c|c|c|c|c|c|c|}
\hline \multirow[b]{2}{*}{ Sex, n (\%) } & \multirow[t]{2}{*}{ New MDD $(\mathrm{N}=287)$} & \multirow[t]{2}{*}{ Remitted MDD $(\mathrm{N}=235)$} & \multicolumn{2}{|l|}{ Non-remitted MDD $(\mathrm{N}=289)$} & p-value & \multirow[t]{2}{*}{ Test $^{\mathrm{a}}$} \\
\hline & & & & & & \\
\hline Male & 87 (30.3) & $60(25.5)$ & $84(29.1)$ & & VS & \\
\hline Female & $200(69.7)$ & $175(74.5)$ & $205(70.9)$ & & & \\
\hline Age (years) & 44.413 .6 & 47.611 .8 & 44.813 .3 & 0.0111 & b & $a=c<b$ \\
\hline \multicolumn{7}{|l|}{ Marital status, n (\%) } \\
\hline Single & $63(22.0)$ & $36(15.3)$ & $62(21.5)$ & & VS & \\
\hline Married & $184(64.1)$ & $165(70.2)$ & $182(63.0)$ & & & \\
\hline Bereaved & $11(3.8)$ & $12(5.1)$ & $21(7.3)$ & & & \\
\hline Divorced & $24(8.4)$ & $15(6.4)$ & $18(6.2)$ & & & \\
\hline Separated & $4(1.4)$ & $7(3.0)$ & $6(2.1)$ & & & \\
\hline \multicolumn{7}{|l|}{ Education, n (\%) } \\
\hline Elementary school & $40(13.9)$ & $32(13.6)$ & $46(15.9)$ & & VS & \\
\hline Middle school & $51(17.8)$ & $31(13.2)$ & $38(13.1)$ & & & \\
\hline High school & $107(37.3)$ & $105(44.7)$ & $121(41.9)$ & & & \\
\hline University & $81(28.2)$ & $56(23.8)$ & $72(24.9)$ & & & \\
\hline Graduate school & $7(2.4)$ & $11(4.7)$ & $12(4.2)$ & & & \\
\hline \multicolumn{7}{|l|}{ Job status, n (\%) } \\
\hline Employed & $128(44.6)$ & $100(42.6)$ & $109(37.7)$ & & VS & \\
\hline Unemployed & $159(55.4)$ & $135(57.4)$ & $180(62.3)$ & & & \\
\hline Onset of MDD (years) & 41.614 .2 & 44.712 .6 & 41.314 .2 & 0.0111 & $\mathrm{~b}$ & $a=c<b$ \\
\hline Number of depressive episodes & 1.12 .0 & 1.32 .2 & 1.72 .6 & 0.0131 & b & $a=b, b=c, a<c$ \\
\hline Duration of depression (months) & 6.313 .7 & 7.316 .6 & 8.120 .4 & NS & & \\
\hline
\end{tabular}

MDD = major depressive disorder; NS = not significant.

${ }^{\text {a }}$ Tukeys post-hoc test.

${ }^{b}$ Difference among MDD groups (ANOVA).

Note: Data are represented as mean ? standard deviation or number of patients (\%). 
Table 2 Severity of depressive and somatic symptoms

\begin{tabular}{|c|c|c|c|c|c|c|}
\hline & New MDD $(\mathrm{N}=287)$ & Remitted MDD ( $\mathrm{N}=235)$ & Unremitted MDD $(\mathrm{N}=289)$ & p-va & & Test $^{a}$ \\
\hline HAM-D scores & 17.87 .0 & 4.52 .1 & 16.65 .8 & $<0.0001$ & b & $\mathrm{b}<\mathrm{c}<\mathrm{a}$ \\
\hline Bech scores ${ }^{c}$ & 8.13 .5 & 2.01 .6 & 7.52 .9 & $<0.0001$ & b & $\mathrm{b}<\mathrm{c}<\mathrm{a}$ \\
\hline Maier-Philipp scores ${ }^{d}$ & 8.23 .7 & 1.81 .5 & 7.43 .2 & $<0.0001$ & b & $\mathrm{b}<\mathrm{c}<\mathrm{a}$ \\
\hline Gibbons scores ${ }^{e}$ & 10.74 .6 & 2.51 .7 & 10.04 .1 & $<0.0001$ & b & $\mathrm{b}<\mathrm{c}=\mathrm{a}$ \\
\hline Suicidality scores & 9.38 .3 & 6.97 .4 & 10.88 .8 & 0.0008 & b & $a=b, a=c, b<c$ \\
\hline DS score & 17.47 .2 & 5.95 .3 & 16.87 .2 & $<0.0001$ & b & $a=c<b$ \\
\hline SS score & 10.95 .8 & 4.03 .9 & 10.66 .2 & $<0.0001$ & b & $a=c<b$ \\
\hline PS score & 5.23 .1 & 2.22 .2 & 5.13 .4 & $<0.0001$ & b & $a=c<b$ \\
\hline DSSS total score & 28.211 .8 & 9.98 .5 & 27.312 .3 & $<0.0001$ & b & $a=c<b$ \\
\hline
\end{tabular}

MDD = major depressive disorder; HAM-D = Hamilton depression rating scale; DSSS = depression and somatic symptoms scale; DS = depression subscale of the DSSS; SS = somatic subscale of the DSSS; PS = pain subscale of the DSSS.

${ }^{\text {a } T u k e y s ~ p o s t-h o c ~ t e s t . ~}$

bDifference among MDD groups (ANOVA).

'Depressed mood, feeling of guilt, work and activities, retardation, anxiety/psychic, and general somatic symptoms are included.

${ }^{\mathrm{d} D e p r e s s e d ~ m o o d, ~ f e e l i n g ~ o f ~ g u i l t, ~ w o r k ~ a n d ~ a c t i v i t i e s, ~ r e t a r d a t i o n, ~ a g i t a t i o n, ~ a n d ~ a n x i e t y / p s y c h i c ~ a r e ~ i n c l u d e d . ~}$

eDepressed mood, feeling of guilt, suicide, work and activities, anxiety/psychic, agitation, anxiety (somatic), and genital symptoms are included.

Note: Data was represented as mean? standard deviation.

anxiety/depression, whereas $87.2 \%$ of the non-remitted group documented some/severe problems. The HRQoL measured by EQ-VAS was comparable to the EQ-5D index score in each group; it was 72.516 .6 in the remitted group and 50.920 .3 in the non-remitted group $(\mathrm{p}<0.0001)$. Life satisfaction measured by Q-LES-Q-SF was approximately 10 points higher in the remitted group than in the non-remitted group (46.5 8.8 vs. 36.7 7.7; $\mathrm{p}<0.0001$ ). More than 95\% of MDD patients were treated with antidepressant medication and $70.8 \%$ of the remitted group were satisfied/very satisfied with their antidepressant medication therapy. However, only $41.8 \%$ of non-remitted patients reported that they were satisfied/very satisfied with their medication.

\section{Relationship between depression subscales and HRQoL}

The results of multiple stepwise regression analysis between the HRQoL and depression subscales are demonstrated in Table 4. When adjusted for age and sex, the symptom severity measured by the DSSS was significantly associated with the HRQoL. The EQ-5D index score decreased by 0.004 in the remitted group $(p=0.0340)$ and 0.017 in the non-remitted group $(\mathrm{p}<0.0001)$ for every unit increase in the somatic symptom score.

Table 5 presents the HRQoL by the level of somatic symptoms in patients over 40 years of age. Depressed adults with severe somatic symptoms $(\geq 8)$ reported a significantly lower EQ-5D index score than those with mild/moderate somatic symptoms $(<8)$. This is shown through the severity of their somatic symptoms: 0.54 0.24 in those with severe symptoms versus 0.750 .12 in those with mild/moderate symptoms $(p=0.0017)$, if adjusted for depression symptoms. Specifically, patients with mild/moderate somatic symptoms showed an EQ-5D index score of 0.720 .14 in the non-remitted group, close to the average EQ-5D index score in the remitted group. Regarding disease-specific HRQoL, patients with severe somatic symptoms presented an approximately $20 \%$ lower mean difference in the Q-LES-Q-SF index score than those with mild/moderate somatic symptoms (0.38 0.14 vs. 0.480 .13$)$.

\section{Association between $\mathrm{HRQ}$ oL and suicidal thoughts/suicide attempts}

Depression severity and impaired HRQoL were significantly associated with an increased risk of suicidal thoughts and suicide attempts (Table 6). Non-remitted patients showed a significantly higher risk of having suicidal thoughts during the past month than those in remission (odds ratio [OR] 2.55; 95\% confidence interval $[\mathrm{CI}], 1.504 .33 ; \mathrm{p}=0.0006)$. Similarly, non-remitted patients showed a significantly higher risk of having made a suicide attempt during the past month than those in remission (OR 2.14; 95\% CI, 1.08 4.25; p = 0.0289). Furthermore, a one-unit improvement in the EQ-5D index score had a $70 \%$ odds reduction of suicidal thoughts (OR 0.3; 95\% CI $0.120 .75 ; \mathrm{p}=0.0104$ ) and a $79 \%$ odds reduction of suicide attempts (OR 0.21; 95\% CI 0.08 0.57; $\mathrm{p}=0.0023)$ in depressed patients.

\section{Discussion}

\section{Overall results}

Here, we examined the HRQoL by the remission status of depressed patients in Korea. In the present study, the nonremitted group showed a significant impairment in HRQoL in view of the subscales of EQ-5D index scores and the EQVAS. Similarly, the life satisfaction measured by the Q-LESQ-SF was significantly lower in the non-remitted group than 
Table 3 HRQoL (EQ-5D, EQ-VAS, Q-LES-Q-SF)

\begin{tabular}{|c|c|c|c|c|c|c|}
\hline \multirow{2}{*}{\multicolumn{7}{|c|}{ New MDD $(\mathrm{N}=287)$ Remitted MDD $(\mathrm{N}=235) \quad$ Non-remitted MDD $(\mathrm{N}=289) \quad \mathrm{p}$-value }} \\
\hline & & & & & & \\
\hline No problems & $246(85.7)$ & $219(93.2)$ & $237(82.0)$ & & & \\
\hline Some problems & $35(12.2)$ & $16(6.8)$ & $49(17.0)$ & & & \\
\hline Severe problems & $6(2.1)$ & $0(0.0)$ & $3(1.0)$ & & & \\
\hline \multicolumn{7}{|l|}{ Self-care, n (\%) } \\
\hline No problems & $268(93.4)$ & $228(97.0)$ & $267(92.4)$ & & & \\
\hline Some problems & $19(6.6)$ & $7(3.0)$ & $21(7.3)$ & & & \\
\hline Severe problems & $0(0.0)$ & $0(0.0)$ & $1(0.3)$ & & & \\
\hline \multicolumn{7}{|l|}{ Usual activity, n (\%) } \\
\hline No problems & $155(54.0)$ & $201(85.5)$ & $143(49.5)$ & & & \\
\hline Some problems & $119(41.5)$ & $33(14.0)$ & $130(45.0)$ & & & \\
\hline Severe problems & $13(4.5)$ & $1(0.4)$ & $16(5.5)$ & & & \\
\hline \multicolumn{7}{|l|}{ Pain/discomfort, n (\%) } \\
\hline No problems & $98(34.1)$ & $153(65.1)$ & $96(33.2)$ & & & \\
\hline Some problems & $163(56.8)$ & 79 (33.6) & $152(52.6)$ & & & \\
\hline Severe problems & $26(9.1)$ & $3(1.3)$ & $41(14.2)$ & & & \\
\hline \multicolumn{7}{|l|}{ Anxiety/depression, n (\%) } \\
\hline No problems & $28(9.8)$ & $143(60.9)$ & $37(12.8)$ & & & \\
\hline Some problems & $189(65.9)$ & $90(38.3)$ & $192(66.4)$ & & & \\
\hline Severe problems & $70(24.4)$ & $2(0.9)$ & $60(20.8)$ & & & \\
\hline EQ-5D index score ${ }^{c}$ & 0.580 .24 & 0.770 .10 & 0.570 .23 & $<0.0001$ & $\mathrm{~b}$ & $\mathrm{a}=\mathrm{c}<\mathrm{b}$ \\
\hline EQ-VAS & 50.320 .6 & 72.516 .6 & 50.920 .3 & $<0.0001$ & $\mathrm{~b}$ & $a=c<b$ \\
\hline Q-LES-Q-SF total score ${ }^{d}$ & 35.67 .8 & 46.58 .8 & 36.77 .7 & $<0.0001$ & $\mathrm{~b}$ & $a=c<b$ \\
\hline Q-LES-Q-SF index score ${ }^{e}$ & 0.390 .14 & 0.580 .16 & 0.410 .14 & $<0.0001$ & b & $a=c<b$ \\
\hline \multicolumn{7}{|c|}{ Status of concomitant medication, n (\%) } \\
\hline Yes & $96(33.4)$ & $226(96.2)$ & $280(96.9)$ & & & \\
\hline No & $191(66.6)$ & $9(3.8)$ & $9(3.1)$ & & & \\
\hline \multicolumn{7}{|c|}{ Satisfaction with medication, n (\%) } \\
\hline Very dissatisfied & $2(2.1)$ & $0(0.0)$ & $5(1.8)$ & & & \\
\hline Dissatisfied & $33(34.4)$ & $14(6.2)$ & $52(18.6)$ & & & \\
\hline Fair & $32(33.3)$ & $52(23.0)$ & $106(37.9)$ & & & \\
\hline Satisfied & $27(28.1)$ & $136(60.2)$ & $106(37.9)$ & & & \\
\hline Very satisfied & $2(2.1)$ & $24(10.6)$ & $11(3.9)$ & & & \\
\hline \multicolumn{7}{|c|}{ Overall life satisfaction and contentment, n (\%) } \\
\hline Very poor & $42(14.6)$ & $1(0.4)$ & $27(9.3)$ & & & \\
\hline
\end{tabular}


Table 3 HRQoL (EQ-5D, EQ-VAS, Q-LES-Q-SF) (Continued)

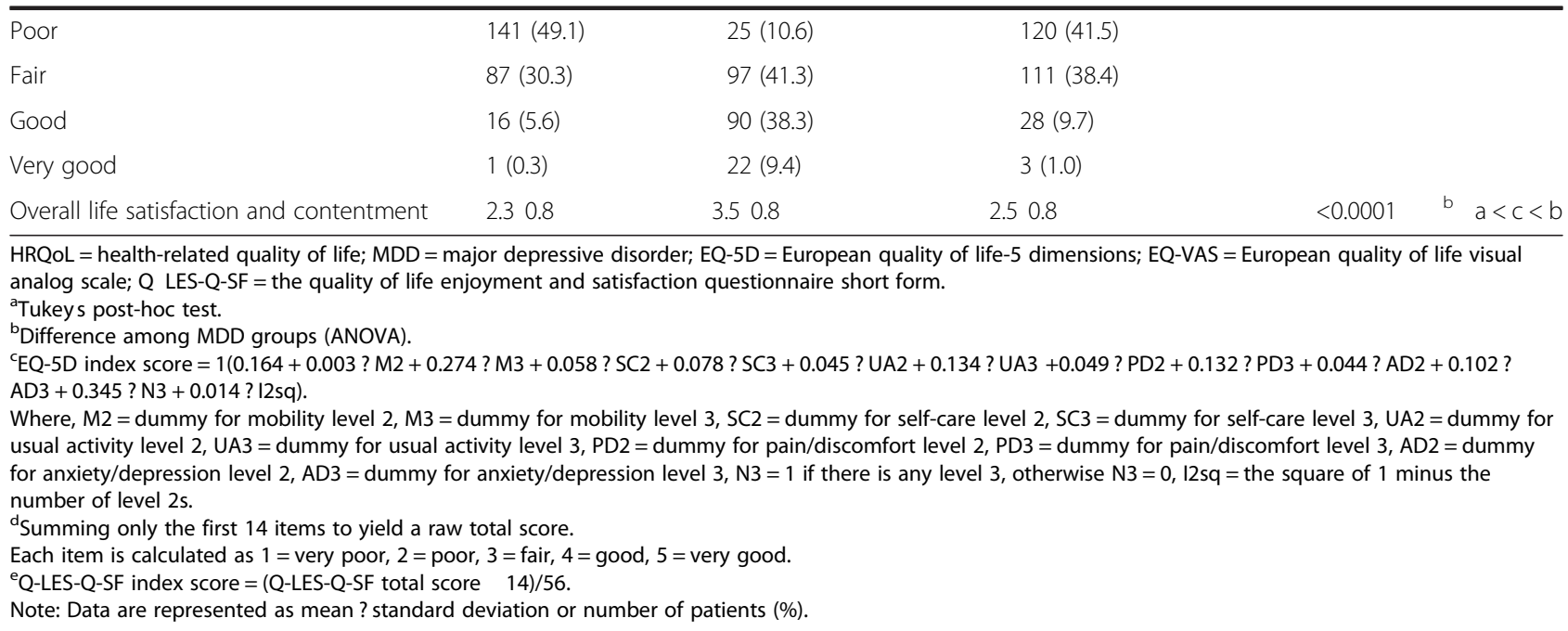

in the remitted group. Even though patients in both groups were treated for their current symptoms of depression, patients who did not reach the treatment goals showed a significantly worse HRQoL than those who met the goals. This finding may suggest that remission achievement in depression plays an important role in the improvement in the preference-based outcomes of patients.

\section{Impact of disease severity on HRQoL}

Previous research in France assessing the HRQoL using the EQ-5D in primary care settings reported EQ-5D index scores of 0.85 for patients in remission, 0.72 for non-remitted patients, and 0.58 for patients who had not responded to the treatment at the 8-week follow-up [28]. Moreover, the FINDER study documenting the impact of antidepressant medication treatment on patients' HRQoL found mean EQ-5D index scores of 0.75 at the 6-month follow-up [8]. Several other studies evaluating HRQoL in depressed patients using various instruments also presented similar utility scores when patients achieved remission: 0.79 measured by McSad [29], 0.70 by quality of well-being [30], and 0.74 by the standard gamble method [31].

In our study, the generic HRQoL measured via the EQ-5D in the remitted group was consistent with the previous research; however, the HRQoL in the nonremitted group was lower than that of previous studies. The EQ-5D index score in our present study was similar to the estimated EQ-5D value of the treatment nonrespondent group in the study conducted by Sapin et al. [28]. There is evidence suggesting that having depression impairs the HRQoL [32] and that antidepressant medication therapy helps to improve HRQoL in depressed patients by decreasing MDD symptoms [8,33]. In our current study, whilst more than $95 \%$ of patients in both the remitted and non-remitted groups were treated with antidepressant medication, satisfaction toward their medication was lowered in the non-remitted group: one of every five patients in the non-remitted group was dissatisfied with their medication use and only $42 \%$ expressed satisfaction in this group. Thus, most patients in the nonremitted group may not respond to their therapy and,

Table 4 Results of stepwise multiple regression analysis: Association between depression and somatic symptoms subscales and the quality of life

\begin{tabular}{|c|c|c|c|c|c|c|c|c|}
\hline \multirow[t]{2}{*}{ Variable } & \multicolumn{2}{|c|}{ New MDD $(\mathrm{N}=287)$} & \multicolumn{2}{|c|}{ Remitted MDD $(\mathrm{N}=235)$} & \multicolumn{2}{|c|}{ Non-remitted MDD $(\mathrm{N}=289)$} & \multicolumn{2}{|c|}{ Total $(\mathrm{N}=811)$} \\
\hline & Coefficient & p-value & Coefficient & p-value & Coefficient & p-value & Coefficient & $p$-value \\
\hline DS score & -0.012 & $<0.0001$ & -0.010 & $<0.0001$ & - & - & -0.008 & $<0.0001$ \\
\hline SS score & -0.006 & 0.0173 & -0.004 & 0.0340 & -0.016 & $<0.0001$ & -0.009 & $<0.0001$ \\
\hline Bech score & -0.012 & 0.0012 & - & - & - & - & - & - \\
\hline Gibbons score & - & - & - & - & -0.017 & $<0.0001$ & -0.008 & $<0.0001$ \\
\hline
\end{tabular}

$\mathrm{MDD}=$ major depressive disorder; DS = depression subscale of the depression and somatic symptoms scale (DSSS); SS = somatic subscale of the DSSS. Stepwise regression analysis using the full set of variables of the DSSS adjusted by age and sex:

Model 1: QoL (EQ-5D index score|New) $=\mathrm{a}+\mathrm{b} 1$ (DS score) + b2(SS score) + b3(Bech) + b4(Age) + b5(Sex)

Model 2: QoL (EQ-5D index score Remitted) $=\mathrm{a}+\mathrm{b} 1$ (DS score) $+\mathrm{b}$ 2(SS score) $+\mathrm{b} 3$ (Age) $+\mathrm{b} 4$ (Sex)

Model 3: QoL (EQ-5D index score|Non-remitted) = a + b1 (SS score) + b2 (Gibbons) + b3 (Age) + b4(Sex). 
Table 5 Quality of life by somatic symptom severity

\begin{tabular}{|c|c|c|c|c|c|c|c|c|c|c|c|c|}
\hline \multirow{3}{*}{$\begin{array}{l}\text { Variable } \\
\text { EQ-5D index score }\end{array}$} & \multicolumn{3}{|l|}{ Overall $^{a}$} & \multicolumn{4}{|c|}{ Remitted MDD } & \multicolumn{5}{|c|}{ Non-remitted MDD } \\
\hline & \multirow{2}{*}{$\begin{array}{l}\mathbf{S S} \geq \mathbf{8} \\
(\mathbf{n}=\mathbf{2 7 7})\end{array}$} & \multirow{2}{*}{$\begin{array}{c}\begin{array}{l}\text { SS }<8 \\
(\mathbf{n}=\mathbf{2 7 9})\end{array} \\
0.750 .12\end{array}$} & $p$-value ${ }^{b}$ & \multirow{2}{*}{$\begin{array}{c}\begin{array}{l}\mathbf{S S} \geq \mathbf{8} \\
(\mathbf{n}=\mathbf{2 8})\end{array} \\
0.650 .19\end{array}$} & \multicolumn{2}{|c|}{$\begin{array}{l}\text { SS }<8 \\
(n=155)\end{array}$} & $p$-value ${ }^{b}$ & \multicolumn{2}{|c|}{$\begin{array}{l}S S \geq 8 \\
(n=118)\end{array}$} & \multicolumn{2}{|c|}{$\begin{array}{l}\text { SS }<8 \\
(n=67)\end{array}$} & \multirow[t]{2}{*}{ p-value ${ }^{b}$} \\
\hline & & & $0.0017^{*}$ & & 0.790 .07 & $0.0139^{*}$ & 0.52 & 0.24 & 0.72 & 0.14 & $0.0015^{*}$ & \\
\hline EQ-VAS & 49.3220 .57 & 69.0317 .56 & $0.0003^{*}$ & 61.2915 .69 & 75.5016 .18 & $0.0372^{*}$ & 47.79 & 920.68 & 59.67 & 16.91 & 0.2218 & \\
\hline Q LES-Q-SF index score & 0.380 .14 & 0.550 .15 & $0.0008^{*}$ & 0.480 .13 & 0.600 .16 & 0.9300 & 0.38 & 0.14 & 0.49 & 0.12 & 0.2973 & \\
\hline
\end{tabular}

$\mathrm{MDD}=$ major depressive disorder; $\mathrm{SS}=$ somatic subscale of the depression and somatic symptoms scale; EQ-5D = European quality of life-5 dimensions; EQ-VAS = European quality of life visual analog scale; $Q$ LES-Q-SF = the quality of life enjoyment and satisfaction questionnaire short form.

${ }^{\text {aP }}$ atients aged 40 years or older.

${ }^{\mathrm{b}}$ The difference between groups was derived from ANCOVA adjusted for depression symptom scores.

*Significance level $\mathrm{p}<0.05$.

therefore, their HRQoL is comparable to the value reported for non-responders.

\section{Impact of somatic symptoms on HRQoL}

Residual somatic symptoms could be another possible explanation for the worse HRQoL in the non-remitted group compared with those of other studies. In our subset analyses, after controlling for depression symptom scores, patients with severe somatic symptoms had significantly worse HRQoL than those who had mild/moderate somatic symptoms. Even non-remitted patients with mild/ moderate somatic symptoms showed comparable EQ-5D index scores to those in remission. This finding indicates that residual somatic symptoms highly influence our patient-reported outcomes.

Several investigators have reported that the presence of somatic symptoms, including painful physical symptoms (PPS), is associated with greater depression severity and poor HRQoL [33-35]. Fava et al. [33] examined the effect of antidepressant medication on PPS in depressed patients, demonstrating that improvements in the PPS were linked to higher remission rates. Other studies showing the association between PPS and HRQoL suggested that patients with positive PPS at the baseline presented 10\% 15\% lower HRQoL scores (EQ-VAS of 42.2 43.0) than those with negative PPS (EQ-VAS of 52.9 60.4) [34,35]. The presence of PPS is still

Table 6 Association between suicidal thoughts/suicide attempts and disease severity and HRQoL

\begin{tabular}{|c|c|c|}
\hline Variable & $\begin{array}{l}\text { Suicidal thoughts } \\
\mathrm{OR}^{\mathrm{a}}(95 \% \mathrm{Cl})\end{array}$ & $\begin{array}{l}\text { Suicide attempts } \\
\mathrm{OR}^{\mathrm{a}}(95 \% \mathrm{Cl})\end{array}$ \\
\hline \multicolumn{3}{|c|}{ Groups by depression severity } \\
\hline Remitted & Reference & Reference \\
\hline Non-remitted & 2.55 (1.50 4.33) & $2.14(1.08 \quad 4.25)$ \\
\hline New visit & 1.56 (0.90 2.70) & $1.21(0.592 .47)$ \\
\hline EQ-5D index score & $0.30(0.120 .75)$ & $0.21(0.080 .57)$ \\
\hline
\end{tabular}

EQ-5D = European quality of life-5 dimensions; OR = odds ratio;

$\mathrm{Cl}=$ confidence interval.

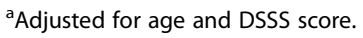

associated with less improvement in HRQoL when adjusted for depression severity $[1,36]$.

Co-existing pain in depressed patients is highly prevalent and more likely to be underdiagnosed and undertreated because of the presence of the depression [37]. The prevalence of co-existing PPS in depressed patients ranges from $43 \%$ to $73 \%$, varying by country [35,38-40]. In particular, $52 \%$ of patients with depression in Asia reported having positive PPS [35]. Despite this awareness, more than $80 \%$ of patients suffering from PPS were not prescribed concomitant treatment for their pain management [35]. Interestingly, the health condition with the largest negative impact on the HRQoL in the general population is pain, followed by depression, osteoarthritis, and anxiety [32]. Due to depressive symptoms or co-existing somatic symptoms, including PPS, depressed patients may be at higher risk of an impaired HRQoL. Therefore, physicians or other health care providers working with depressed patients should pay careful attention to somatic symptoms as well as classical depressive symptoms if such patients are seeking help regarding their depression.

\section{Comparison between preference-based and disease- specific HRQoL}

In our present study, subjects showed lower Q-LES-Q-SF scores as disease-specific QoL measures than those of previous studies. The scores were $46.5 \%$ in the remitted group and $36.7 \%$ in the non-remitted group, whereas previous studies reported Q-LES-Q-SF scores of 52\% $54 \%$ in patients with depression and $74 \% 79 \%$ in subjects that had once had depression $[41,42]$. The discrepancy may be related to the different levels of severity between the studies. Previous investigations assessed the disease-specific QoL in patients with chronic major depression or dysthymia, whereas it was assessed in subjects with more severe depression in university hospital settings in our study.

\section{HRQoL and suicidal thoughts/suicide attempts}

In general, QoL is measured on a scale from 0 (deceased) to 1 (completely healthy), indicating that death is the worst state that people can imagine [19]. An impaired HRQoL is also significantly associated with both suicidal 
thoughts and suicide attempts [43]. One study using a population-based data survey showed that people with an EQ-5D index score less than 0.7 were 3.4 times more likely to attempt suicide than those with an index score of 0.81 .0 [43]. This study also reported that people with an EQ-5D index score less than 0.7 had a 9.1 times increased risk of suicidal thoughts than those with a score between 0.8 1.0. Our study found a comparable pattern of association in patients with depression. Non-remitted patients with a worse HRQoL showed a more than 2-fold increased risk of suicidal thoughts and suicide attempts than those in remission. Furthermore, the improvement in EQ-5D index scores significantly reduced both suicidal thoughts and suicide attempts in depressed patients. Our findings indicate the importance of achieving remission in the treatment of depressed patients, not only for the improvement of HRQoL, but also for the prevention of the worst outcomes, such as suicide or death.

\section{Strengths and limitations of the study}

Our study has several limitations. First, due to the nature of a cross-sectional study, we were unable to address the improvement in HRQoL according to the type of treatment or total duration. However, our investigation involved multiple sites in Korea, reflecting a similar cohort to a real world population with depression. Furthermore, by recruiting the new visit group, we attempted to show the initial baseline characteristics of patients who visited the hospitals. Second, the depression treatment was provided based on the health care delivery system of each site. The possible differences between sites might have affected our HRQoL results. To minimize unintended bias, we recruited the same number of patients from each site.

\section{Conclusions}

We evaluated the HRQoL by disease severity in patients with depression. Non-remitted MDD patients with more severe somatic symptoms suffer from a poorer HRQoL and have more clinical symptoms, emphasizing the importance of achieving remission in the treatment of subjects with MDD in order to improve their HRQoL. In addition, the presence of somatic symptoms is significantly associated with impairment of HRQoL, suggesting that somatic symptoms of patients should be carefully evaluated to improve the preference-based QoL.

\footnotetext{
Abbreviations

DS: Depression subscale of the depression and somatic symptoms scale; DSSS: The depression and somatic symptoms scale; EQ-5D: European quality of life-5 dimensions; EQ-VAS: European quality of life visual analog scale; HAM-D: Hamilton depression rating scale; HAM-D-17: 17 items Hamilton depression rating scale; HRQoL: Health-related quality of life; MDD: Major depressive disorder; MINI: The mini-international neuropsychiatric interview; PS: Pain subscale of the depression and somatic symptoms scale; Q LES-Q: The quality of life enjoyment and satisfaction questionnaire; Q LES-Q-SF: the quality of life enjoyment and satisfaction questionnaire short form; QoL: Quality of life; SS: Somatic subscale of the depression and somatic symptoms scale.
}

\section{Competing interests}

Jong-Min Woo, Hong Jin Jeon, Sun Woo Lee, Kyung Kyu Lee, Sung Hwan Kim, and Jin Pyo Hong received research funding from Pfizer Inc. Eunsun Noh has no conflicts to disclose. Hyo-Jin Kim is a Pfizer employee; however, there are no conflicts to disclose regarding this research.

\section{Authors contributions}

JW designed the study, performed the analyses, interpreted the results, and drafted the manuscript. HJ conceived the study and collected the data. EN provided analysis advice and strongly contributed to the manuscript. HK participated in the design of the study and performed the statistical analysis. $\mathrm{SL}, \mathrm{KL}$, and $\mathrm{SJ}$ collected the data. JH is corresponding author and critically appraised the manuscript. All authors read and approved the final manuscript.

\section{Acknowledgements}

This study was supported by Pfizer Pharmaceuticals Korea Ltd. and by the Basic Science Research Program through the National Research Foundation of Korea (NRF) of the Ministry of Education, Science and Technology (No. 2011-0013064)

\section{Author details}

${ }^{1}$ Department of Psychiatry, Seoul Paik Hospital, Inje University School of Medicine, Seoul, Korea. ${ }^{2}$ Stress Research Institute, Inje University, Seoul, Korea. ${ }^{3}$ Department of Psychiatry, Depression Center, Samsung Medical Center, Sungkyunkwan University School of Medicine, Seoul, Korea. ${ }^{4}$ Depression Clinical and Research Program, Massachusetts General Hospital, Harvard Medical School, Boston, USA. ${ }^{5}$ Veterans Affairs Medical Center, Providence, Rhode Island, USA. ${ }^{6}$ Department of Pharmacy Practice, College of Pharmacy, University of Rhode Island, Kingston, Rhode Island, USA. ${ }^{7}$ OR/RWD Team, Health and Value Division, Pfizer Pharmaceuticals Korea Limited, Seoul, Korea. ${ }^{8}$ Department of Psychiatry, Chungnam National University School of Medicine, Chungnam, Korea. ${ }^{9}$ Department of Psychiatry, Dankook University School of Medicine, Cheonan, Korea. ${ }^{10}$ Department of Psychiatry, Dong-A University School of Medicine, Busan, Korea. ${ }^{11}$ Department of Psychiatry, Asan Medical Center, Ulsan University College of Medicine, 388-1

Pungnap-2dong, Songpa-gu, Seoul 138-736, South Korea.

Received: 11 September 2014 Accepted: 8 December 2014

Published online: 18 December 2014

\section{References}

1. Caruso R, Rossi A, Barraco A, Quail D, Grassi L, group IFs: The Factors Influencing Depression Endpoints Research (FINDER) study: final results of Italian patients with depression. Ann Gen Psychiatry 2010, 9:33.

2. Moussavi S, Chatterij S, Verdes E, Tandon A, Patel V, Ustun B: Depression, chronic diseases, and decrements in health: results from the World Health Surveys. Lancet 2007, 370:851 858.

3. Papakostas Gl, Petersen T, Mahal Y, Mischoulon D, Nierenberg AA, Fava M: Quality of life assessments in major depressive disorder: a review of the literature. Gen Hosp Psychiatry 2004, 26:13 17.

4. Cramer JA, Rosenheck R, Xu W, Thomas J, Henderson W, Dennis SC: Quality of Life in Schizophrenia: A Comparison of Instruments. Department of Veterans Affairs Cooperative Study Group on Clozapine in Refractory Schizophrenia. Schizophr Bull 2000, 26:659 666.

5. Coons SJ, Gwaltney CJ, Hays RD, Lundy JJ, Sloan JA, Revicki DA, Lenderking WR, Cella D, Basch E: Recommendations on Evidence Needed to Support Measurement Equivalence between Electronic and Paper-Based Patient-Reported Outcome (PRO) Measures: ISPOR ePRO Good Research Practices Task Force Report. Value Health 2009, 12:419 429 .

6. U.S. Department of Health and Human Services FDA Center for Drug Evaluation and Research, U.S. Department of Health and Human Services FDA Center for Biologics Evaluation and Research, U.S. Department of Health and Human Services FDA Center for Devices and Radiological Health: Guidance for industry: patient-reported outcome measures: use in medical product development to support labeling claims: draft guidance. Health Qual Life Outcomes 2006, 4:79.

7. Garca-Campayo J, Ayuso-Mateos JL, Caballero L, Romera I, Aragons E, Rodrguez-Artalejo F, Quail D, Gilaberte I: Relationship of somatic symptoms with depression severity, quality of life, and health resources 
utilization in patients with major depressive disorder seeking primary health care in Spain. Prim Care Companion J Clin Psychiatry 2008, 10:355 362 .

8. Reed C, Monz BU, Perahia DGS, Gandhi P, Bauer M, Dantchev N, Demyttenaere K, Garcia-Cebrian A, Grassi L, Quail D, Tylee A, Montejo AL: Quality of life outcomes among patients with depression after 6 months of starting treatment: Results from FINDER. J Affect Disord 2009, 113:296 302.

9. Judd LL, Akiskal HS, Maser JD, Zeller PJ, Endicott J, Coryell W, Paulus MP, Kunovac JL, Leon AC, Mueller TI, Rice JA, Keller MB: Major depressive disorder: A prospective study of residual subthreshold depressive symptoms as predictor of rapid relapse. J Affect Disord 1998, 50:97 108.

10. Hamilton M: Development of a Rating Scale for Primary Depressive Illness. Br J Soc Clin Psychol 1967, 6:278 296

11. Frank E, Prien RF, Jarrett RB, Keller MB, Kupfer DJ, Lavori PW, Rush AJ, Weissman MM: Conceptualization and rationale for consensus definitions of terms in major depressive disorder: Remission, recovery, relapse, and recurrence. Arch Gen Psychiatry 1991, 48:851 855.

12. Rush AJ, Trivedi MH, Ibrahim HM, Carmody TJ, Arnow B, Klein DN, Markowitz JC, Ninan PT, Kornstein S, Manber R, Thase ME, Kocsis JH, Keller $M B$ : The 16-Item quick inventory of depressive symptomatology (QIDS), clinician rating (QIDS-C), and self-report (QIDS-SR): a psychometric evaluation in patients with chronic major depression. Biol Psychiatry 2003, 54:573 583.

13. Kobak KA, Lipsitz JD, Feiger A: Development of a standardized training program for the Hamilton Depression Scale using internet-based technologies: results from a pilot study. J Psychiatr Res 2003, 37:509 515.

14. Williams JW: A structured interview guide for the hamilton depression rating scale. Arch Gen Psychiatry 1988, 45:742 747

15. Hung Cl, Wang SJ, Liu CY: Validation of the Depression and Somatic Symptoms Scale by comparison with the Short Form 36 scale among psychiatric outpatients with major depressive disorder. Depress Anxiety 2009, 26:583 591.

16. Kim KW, Hong JP, Park SJ, Choi JH, Choi HR: Reliability and Validity of Korean Version of Depression and Somatic Symptom Scale(DSSS). Anxiety and Mood 2011, 7:9 15.

17. Kim KY, Chun BY, Kam S, Lee SW, Park KS, Chae SC: [Development of measurement scale for the quality of life in hypertensive patients]. J Prev Med Public Health 2005, 38:61 70.

18. Brazier J: Measuring and valuing mental health for use in economic evaluation. J Health Serv Res Policy 2008, 13(Suppl 3):70 75.

19. Brazier J: Is the EQ $5 \mathrm{D}$ fit for purpose in mental health? Br J Psychiatry 2010, 197:348 349 .

20. Group EQ: EuroQol a new facility for the measurement of health-related quality of life. Health Policy 1990, 16:199 208.

21. Kim M-H, Cho Y-S, Uhm W-S, Kim S, Bae S-C: Cross-cultural adaptation and validation of the Korean version of the EQ-5D in patients with rheumatic diseases. Qual Life Res 2005, 14:1401 1406.

22. Kang EJ, Shin HS, Park HJ, Cho MW, Kim NY: A Valuing health states using EQ-5D. Seoul: Management Center for Health Promotion, Korea Institute for Health and Social Affairs; 2006.

23. Endicott J, Nee J, Harrison W, Blumenthal R: Quality of Life Enjoyment and Satisfaction Questionnaire: a new measure. Psychopharmacol Bull 1993, 29:321 326.

24. Wisniewski SR, Rush AJ, Bryan C, Shelton R, Trivedi MH, Marcus S, Husain MM, Hollon SD, Fava M, Investigators ftS: Comparison of Quality of Life Measures in a Depressed Population. J Nerv Ment Dis 2007, 195:219 225.

25. Schechter D, Endicott J, Nee J: Quality of life of normal controls: Association with lifetime history of mental illness. Psychiatry Res 2007, 152:45 54.

26. Testa MA, Simonson DC: Assessment of quality-of-life outcomes. N Engl J Med 1996, 334:835 840.

27. Sheehan DV, Lecrubier Y, Sheehan KH, Amorim P, Janavs JW E, Hergueta T, Baker R, Dunbar GC: The Mini-International Neuropsychiatric Interview (M.I.N.I.): the development and validation of a structured diagnostic psychiatric interview for DSM-IV and ICD-10. J Clin Psychiatry 1998, 59(Suppl 20):22 33. quiz 34-57.

28. Sapin C, Fantino B, Nowicki M-L, Kind P: Usefulness of EQ-5D in Assessing Health Status in Primary Care Patients with Major Depressive Disorder. Health Qual Life Outcomes 2004, 2:20.
29. Bennett KJ, Torrance GW, Boyle MH, Guscott R, Moran LA: Development and Testing of a Utility Measure for Major, Unipolar Depression (McSad). Qual Life Res 2000, 9:109 120.

30. Pyne JM, Patterson TL, Kaplan RM, Gillin JC, Koch WL, Grant I: Assessment of the quality of life of patients with major depression. Psychiatr Serv 1997, 48:224 230.

31. Revicki DA, Wood M: Patient-assigned health state utilities for depression-related outcomes: differences by depression severity and antidepressant medications. J Affect Disord 1998, 48:25 36.

32. Wu M, Brazier J, Kearns B, Relton C, Smith C, Cooper C: Examining the impact of 11 long-standing health conditions on health-related quality of life using the EQ-5D in a general population sample. Eur J Health Econ. in press.

33. Fava M, Mallinckrodt $\mathrm{CH}$, Detke MJ, Watkin JG, Wohlreich MM: The effect of duloxetine on painful physical symptoms in depressed patients: do improvements in these symptoms result in higher remission rates? J Clin Psychiatry 2004, 65:521 530.

34. Bahk WM, Park S, Jon DI, Yoon BH, Min KJ, Hong JP: Relationship between painful physical symptoms and severity of depressive symptomatology and suicidality. Psychiatry Res 2011, 189:357 361

35. Lee $P$, Zhang M, Hong JP, Chua HC, Chen KP, Tang SW, Chan BT, Lee MS, Lee B, Gallagher GL, Dossenbach M: Frequency of painful physical symptoms with major depressive disorder in asia: relationship with disease severity and quality of life. J Clin Psychiatry 2009, 70:83 91.

36. D Avanzato C, Martinez J, Attiullah N, Friedman M, Toba C, Boerescu DA, Zimmerman M: Anxiety symptoms among remitted depressed outpatients: Prevalence and association with quality of life and psychosocial functioning. J Affect Disord 2013, 151:401 404.

37. Katon W, Sullivan MD: Depression and chronic medical illness. J Clin Psychiatry 1990, 51(Suppl):3 11. discussion 12-14.

38. Bair MJ, Robinson RL, Eckert GJ, Stang PE, Croghan TW, Kroenke K: Impact of Pain on Depression Treatment Response in Primary Care. Psychosom Med 2004, 66:17 22

39. Muoz RA, McBride ME, Brnabic AJM, Lpez CJ, Hetem LAB, Secin R, Dueas HJ: Major depressive disorder in Latin America: The relationship between depression severity, painful somatic symptoms, and quality of life. J Affect Disord 2005, 86:93 98.

40. Ohayon MM, Schatzberg AF: USing chronic pain to predict depressive morbidity in the general population. Arch Gen Psychiatry 2003, 60:39 47.

41. Kocsis JH, Zisook S, Davidson J, Shelton R, Yonkers K, Hellerstein DJ, Rosenbaum J, Halbreich U: Double-blind comparison of sertraline, imipramine, and placebo in the treatment of dysthymia: psychosocial outcomes. Am J Psychiatry 1997, 154:390 395

42. Russell JM, Koran LM, Rush J, Hirschfeld RMA, Harrison W, Friedman ES Davis S, Keller M: Effect of concurrent anxiety on response to sertraline and imipramine in patients with chronic depression. Depress Anxiety 2001, 13:18 27.

43. Kwon JW, Kim JH: The impact of health-related quality of life on suicidal ideation and suicide attempts among Korean older adults. J Gerontol Nurs 2012, 38:48 59

\section{doi:10.1186/s12955-014-0188-y}

Cite this article as: Woo et al:: Importance of remission and residual somatic symptoms in health-related quality of life among outpatients with major depressive disorder: a cross-sectional study. Health and Quality of Life Outcomes 2014 12:188.

\section{Submit your next manuscript to BioMed Central and take full advantage of:}

凶Convenient online submission

$\otimes$ Thorough peer review

$\nabla$ No space constraints or color $\nabla$ gure charges

$\otimes I m m e d i a t e$ publication on acceptance

$\triangle$ Inclusion in PubMed, CAS, Scopus and Google Scholar

$\nabla$ Research which is freely available for redistribution 